\title{
Real time monitoring of the suitability of high resolution LC-MS systems by image recognition algorithm using peaks of spiked-in internal standards
}

Serhiy Hnatyshyn ${ }^{1}$, Rostyslav Hnatyshyn ${ }^{2}$, Jan-Lucas Ott ${ }^{1}$

Technical program of the ACS Spring 2020 National Meeting \& Expo,

March 22-26, 2020

1 - Bristol-Myers Squibb Company

2-Rowan University 


\section{Outline}

- Introduction: Data quality in metabolomics experiments

- High resolution LC-MS workflow

- The components of data quality

- System suitability testing procedures

- Chromatographic peaks as quality indicators

- Internal standard (IS): N-Benzoyl-d5-glycine

- Visual inspection of IS peaks

- Logarithm transformation

- Data processing algorithm

- Statistics of analysis of IS peaks

- Peak parameters vs. injection order

- Peak data as $28 \times 28$ pixel image

- Convolution Neural Network (CNN)

- CNN model for peak abnormality detection

- Model prototype implementation using Jupyter Notebook (Python 3)

- CNN model applications for real-time study monitoring examples

- Lessons learned

- Conclusions and future work 


\section{Introduction}

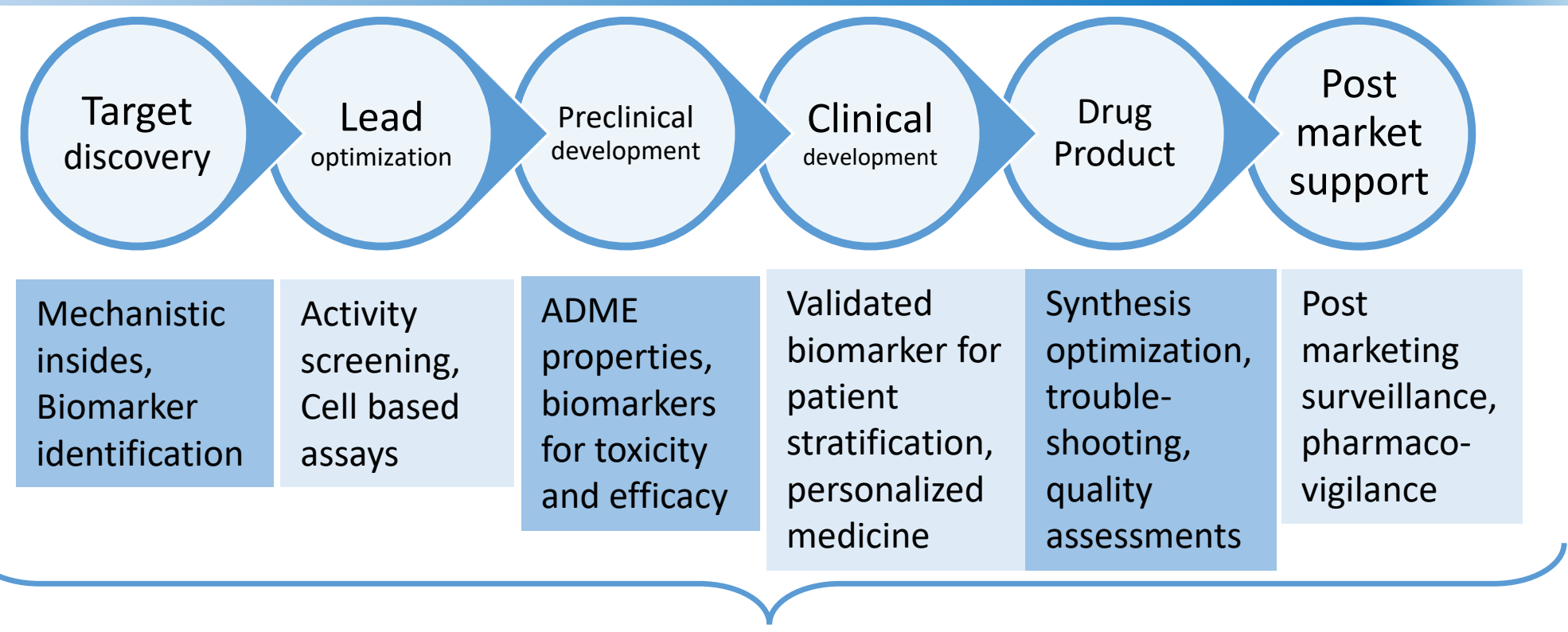

\section{We are most concerned with the qualitative composition of a given sample, as well as its quantitative makeup.}

\section{Data quality is a cornerstone.}

The continuous monitoring of system performance during high resolution LC-MS metabolomics studies is necessary not only for data quality control, but also to maintain productivity and prevent the loss of precious samples. Currently, due to practical constraints, such monitoring is not continuous. Considering the long duration of the experiment - often several days, monitoring is typically performed by introducing internal standards and then examining the peaks associated with the internal standards after data acquisition. Such an approach can overlook abnormal events during the acquisition of the data, which leads to poor data quality or in extreme cases, the need to repeat the run. 


\section{High resolution LC-MS Workflow}

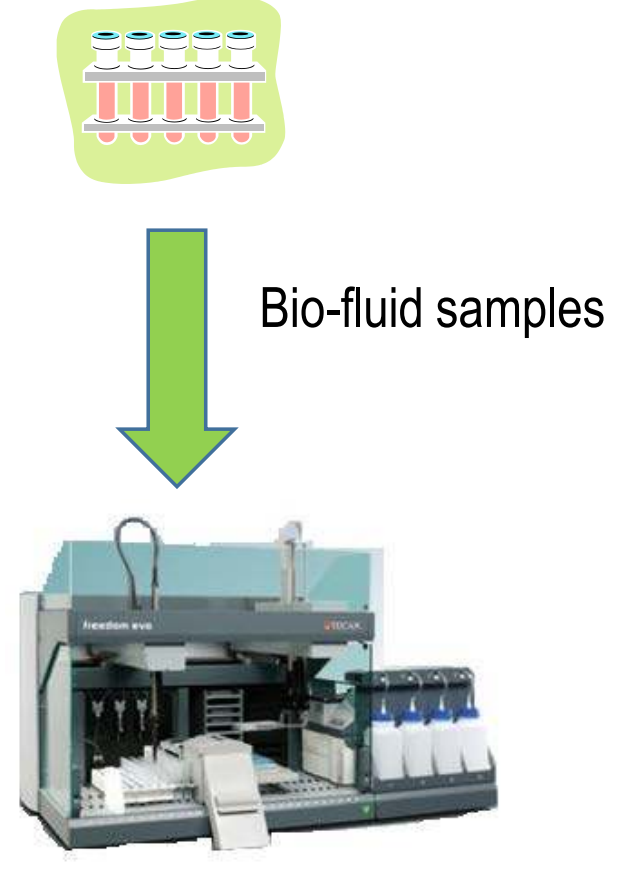

$\sim 400,000,000$ Data points in each polarity $\sim 200 \mathrm{~Gb}$ of disk space per study
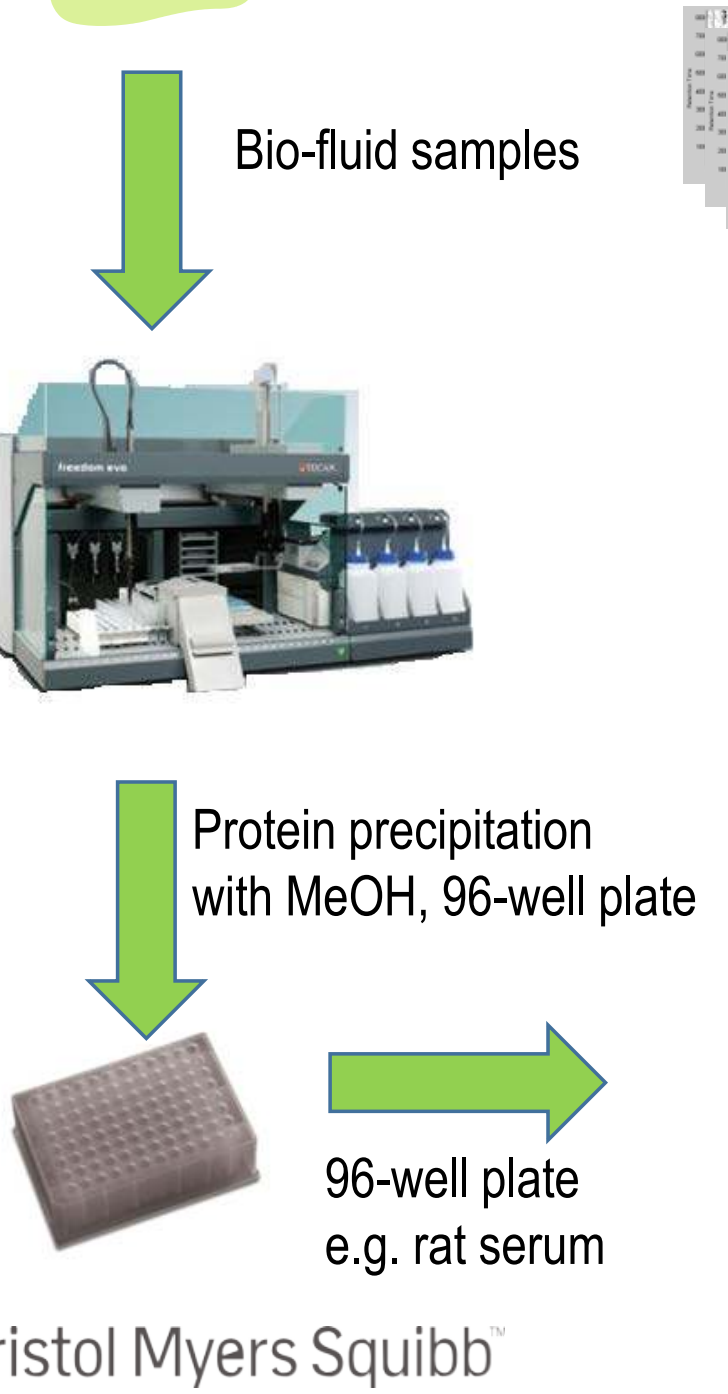
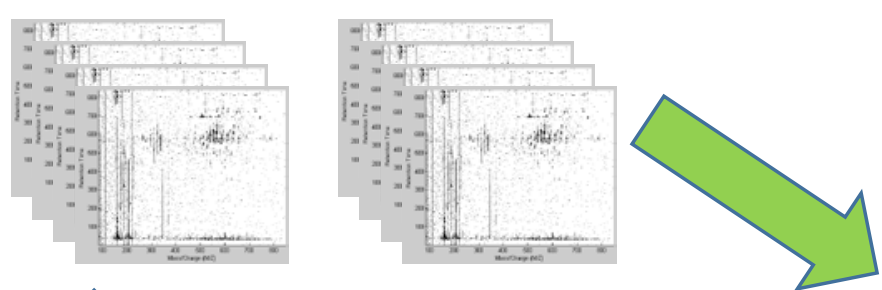

Data reduction and annotation using in-house developed software

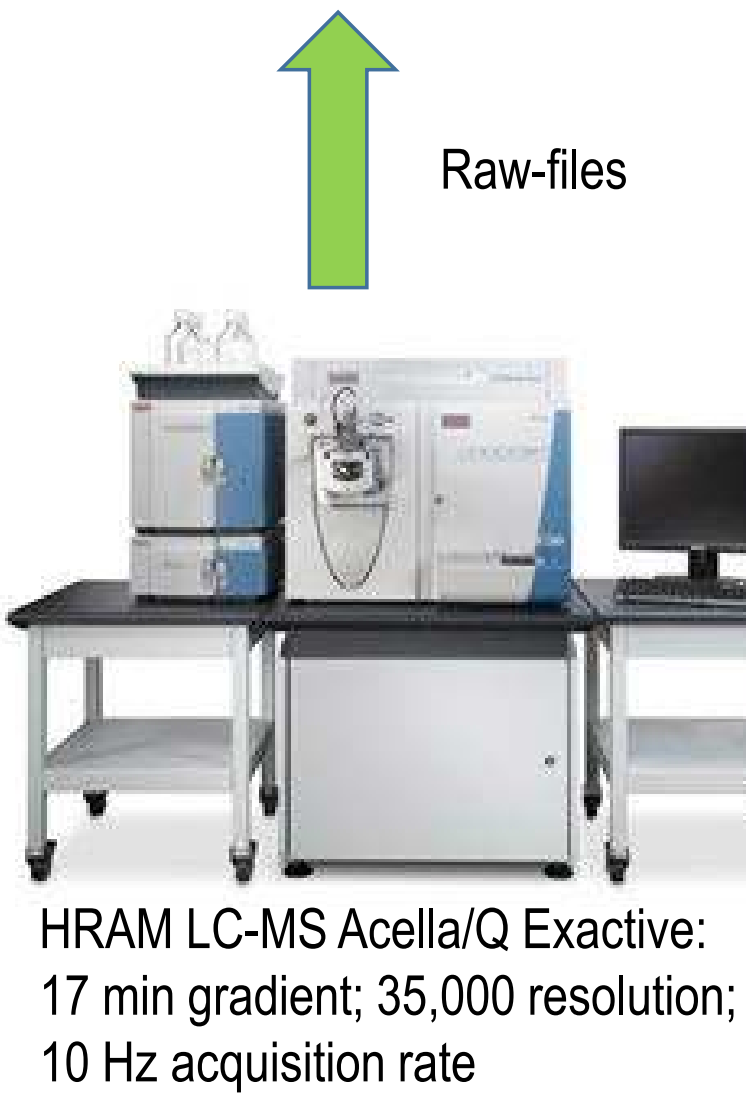

ACS Spring 2020 National Meeting \& Expo 


\section{The Components of Data Quality}

\begin{tabular}{|c|c|c|}
\hline Component & Description & Frequency of usage \\
\hline $\begin{array}{l}\text { Analytical } \\
\text { Instrument } \\
\text { Qualification }\end{array}$ & $\begin{array}{c}\text { Documented evidence that an instrument } \\
\text { performs suitably for its intended purpose } \\
\text { and that the instrument is properly } \\
\text { maintained and calibrated. }\end{array}$ & $\begin{array}{c}\text { Installation, major maintanence } \\
\text { event, relocation }\end{array}$ \\
\hline $\begin{array}{l}\text { Analytical } \\
\text { Method } \\
\text { Validation }\end{array}$ & $\begin{array}{l}\text { A collection of all necessary procedures that } \\
\text { demonstrate that a particular method is } \\
\text { reliable and reproducible. Special } \\
\text { considerations are used for the measurement } \\
\text { of analytes in a biological matrix such as } \\
\text { blood, plasma, serum, or urine. }\end{array}$ & $\begin{array}{l}\text { Method development when } \\
\text { establishing workflow }\end{array}$ \\
\hline $\begin{array}{l}\text { System } \\
\text { Suitability } \\
\text { Testing }\end{array}$ & $\begin{array}{c}\text { Daily test to verify the adequate working of } \\
\text { the equipment used for analytical } \\
\text { measurements. }\end{array}$ & Daily, before testing begin \\
\hline $\begin{array}{l}\text { Quality } \\
\text { Control } \\
\text { Checks }\end{array}$ & $\begin{array}{l}\text { Spiked samples of know quality are used to } \\
\text { monitor the performance of a bioanalytical } \\
\text { method. They are allso used to assess the } \\
\text { integrity and validity of the results of unknown } \\
\text { samples within the same batch. }\end{array}$ & $\begin{array}{l}\text { Every } N \text { (usually } N=10 \text { ) samples } \\
\text { during the batch run }\end{array}$ \\
\hline $\begin{array}{l}\text { Internal } \\
\text { Standards }\end{array}$ & $\begin{array}{c}\text { Spiked labeled compounds to monitor } \\
\text { indiviual sample run }\end{array}$ & Every sample \\
\hline
\end{tabular}

rare

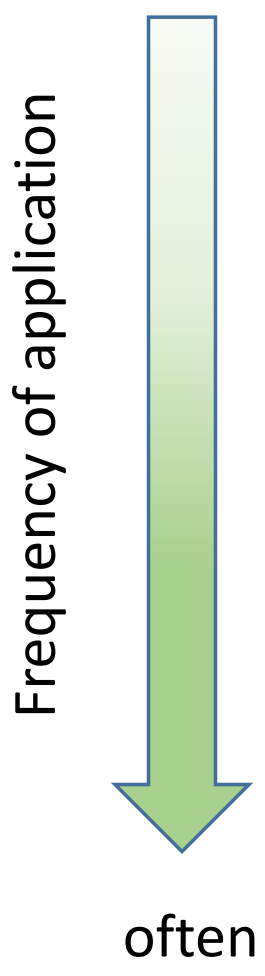

Reference : C.J. Briscoe et al. / Journal of Pharmaceutical and Biomedical Analysis 44 (2007) 484-491 


\section{System suitability testing procedures}

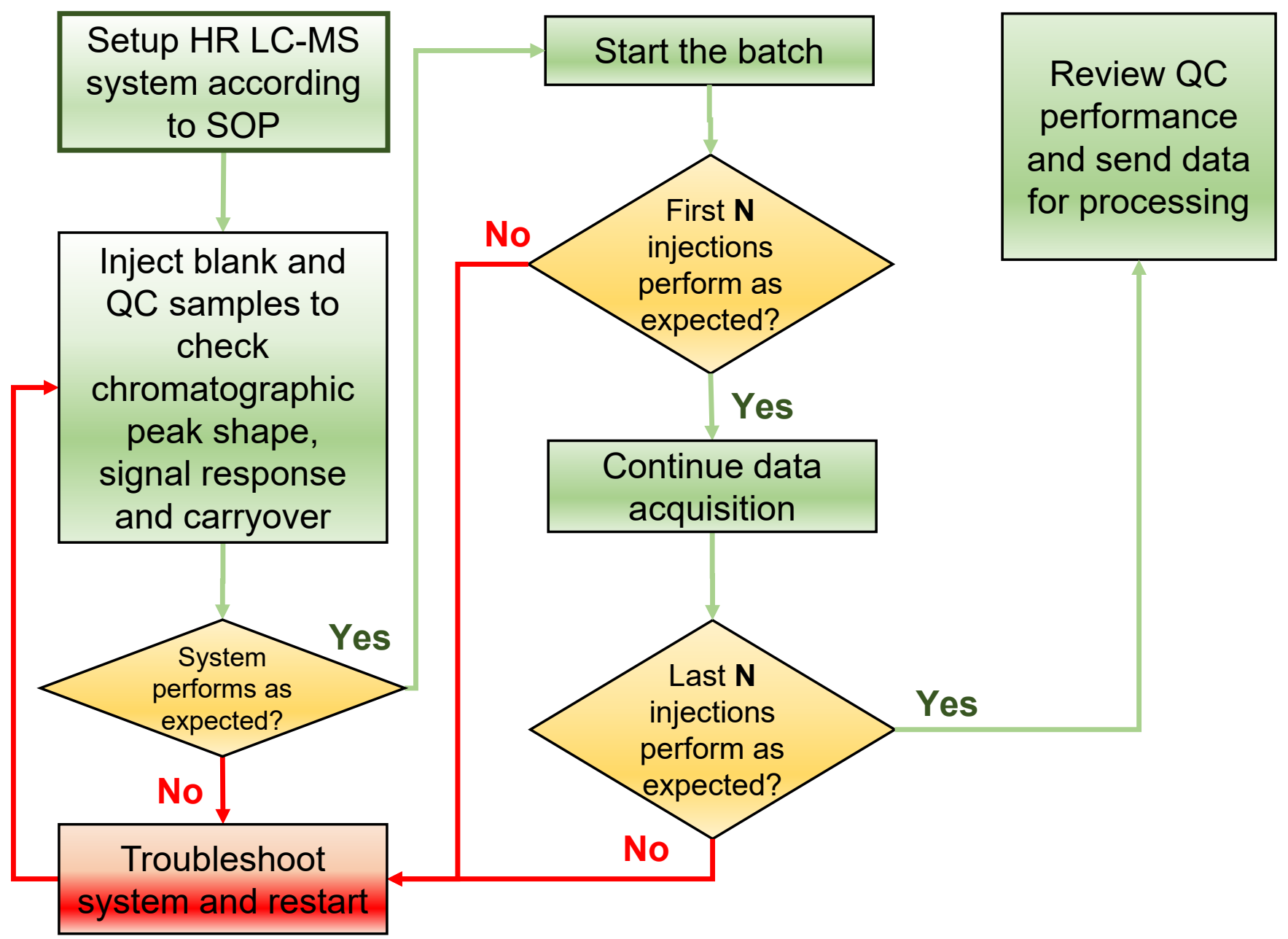

For long runs, it is impractical to check every sample. In practice, spot checks are utilized.

Realizing that the samples are compromised at the processing stage leads to missing data and lost opportunities.

Reference : C.J. Briscoe et al. / Journal of Pharmaceutical and Biomedical Analysis 44 (2007) 484-491 


\section{Chromatographic peaks as quality indicators}

"The significant deviation of the peak shape from the symmetrical peak makes hardly possible the acquisition of chromatographic signal information, such as the retention time, the peak area, the peak width at half peak height, the peak overlapping, etc."

Zs. Pápai, T.L. Pap "Analysis of peak asymmetry in chromatography" Journal of Chromatography A, 953 (2002) 31-38

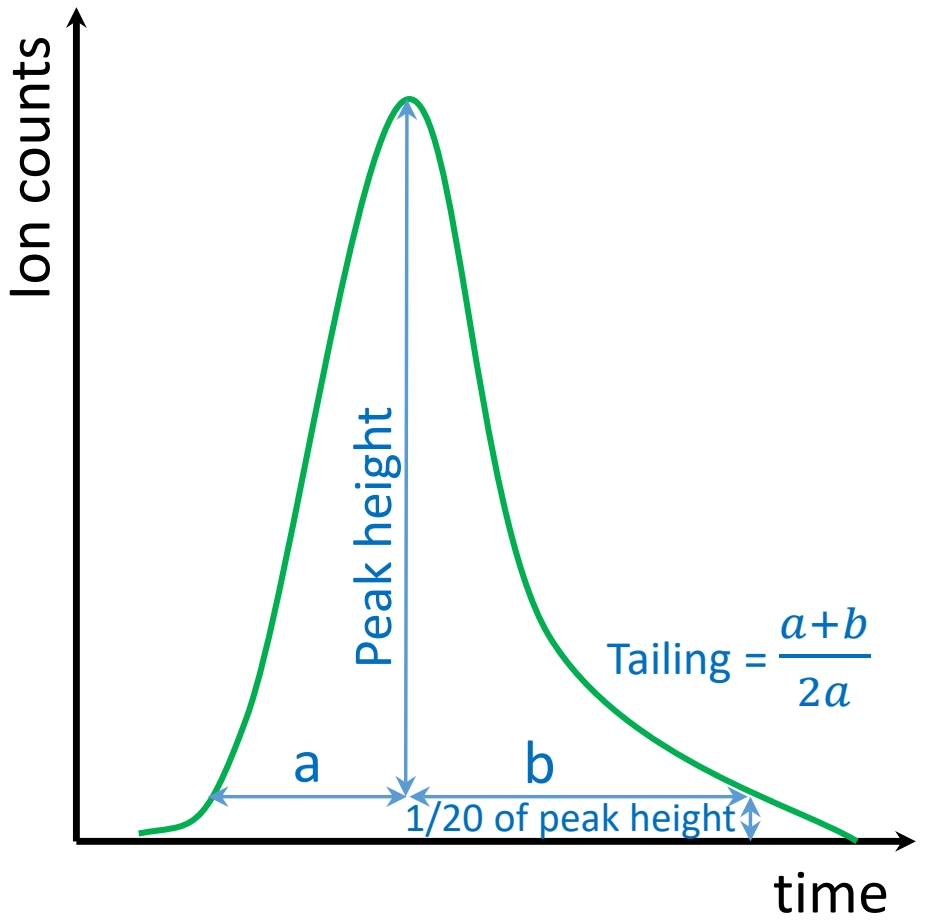

Typical extracted ion chromatogram peak for a "well-behaved" quality control
The trade off between scan rate and mass resolution is defined in the setup of the LC-MS experiment. Both parameters influence the quality of chromatographic peaks.

UPLC provides fast chromatography improving the throughput of the analysis. A typical peak width is 4-6 seconds.

For such narrow peaks, a $Q$ Exactive mass spectrometer operating at 70,000 resolution acquires $>15$ points across the peak without losing sensitivity*.
*Junhua Wang, David A. Peake, Mark Sanders, Michael Athanas, Yingying Huang Untargeted Metabolomics Workflow Using UHPLC/Quadrupole Orbitrap Mass Spectrometer and SIEVE 2.1., Thermo Fisher Scientific Inc, San Jose, CA, USA 


\section{IS: N-Benzoyl-d5-glycine}

\section{Product code: D-5588}

Chemical formula: $\mathrm{C}_{9} \mathrm{H}_{4} \mathrm{D}_{5} \mathrm{NO}_{3}$

CAS: $53518-98-2$

CAS (unlabelled): 495-69-2

Synonyms: Hippuric Acid, Benzoylaminoacetic Acid, Bz-Gly-OH

Supplier / Manufacturer:

C/D/N Isotopes Inc.

88 Leacock Street

Pointe-Claire (Québec) H9R 1H1

Phone: 514-697-6254

Toll-Free (Canada \& USA): 1-800-565-4696 Fax: 514-697-6148

Website: www.cdnisotopes.com<smiles>[2H]c1c([2H])c([2H])c(C(=O)NCC(=O)O)c([2H])c1[2H]</smiles>

d5-Hippuric Acid

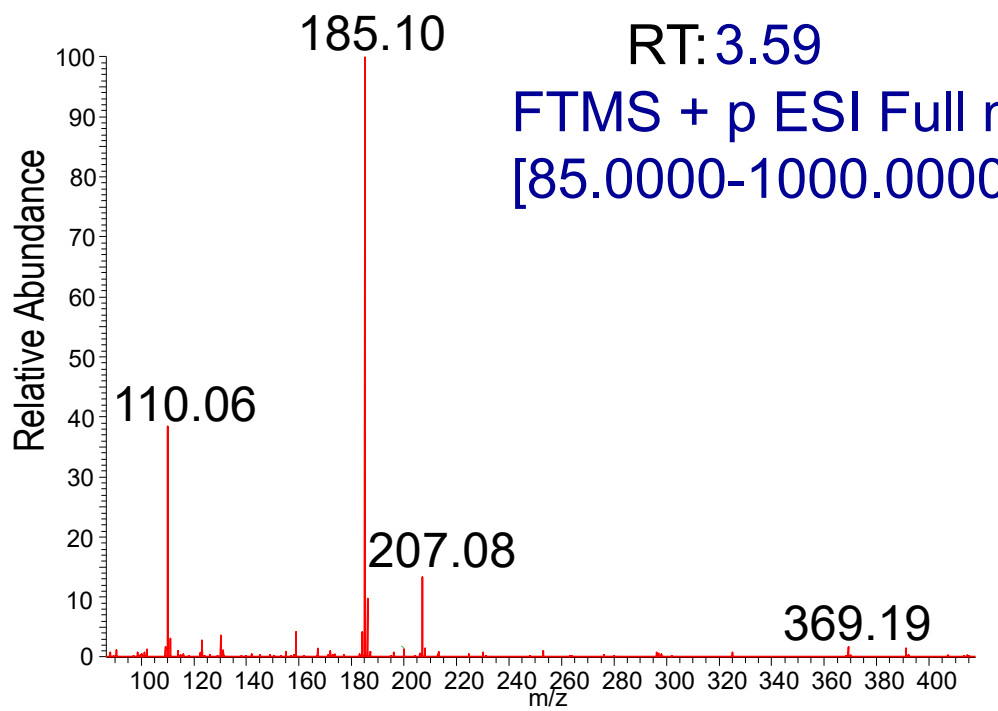

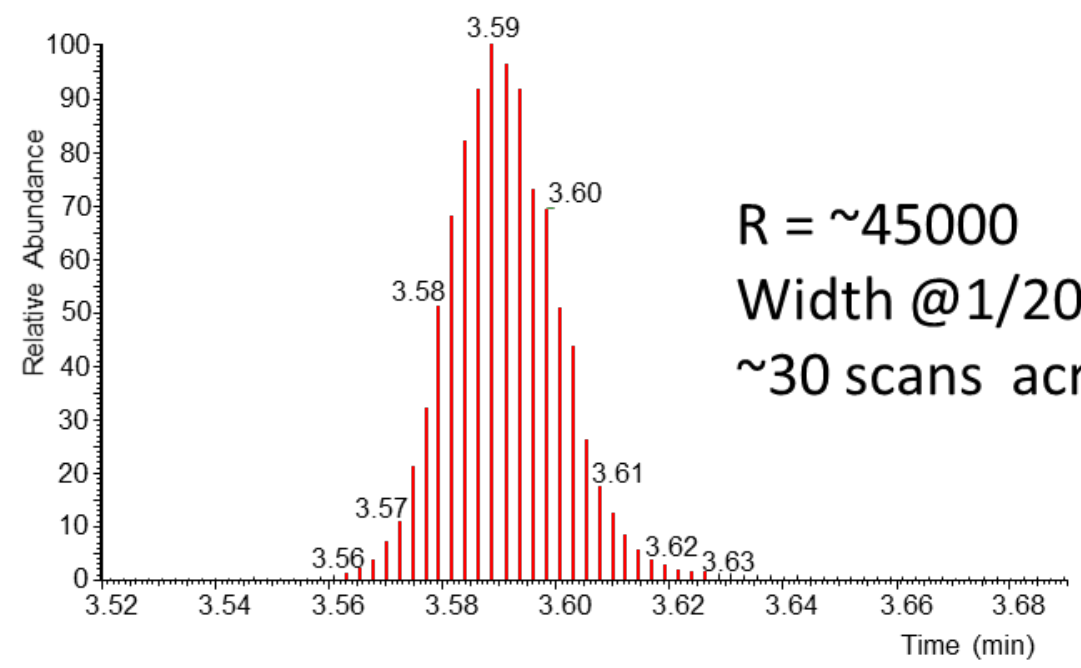

$$
\text { IS = internal standard }
$$




\section{Visual inspection of IS peaks}

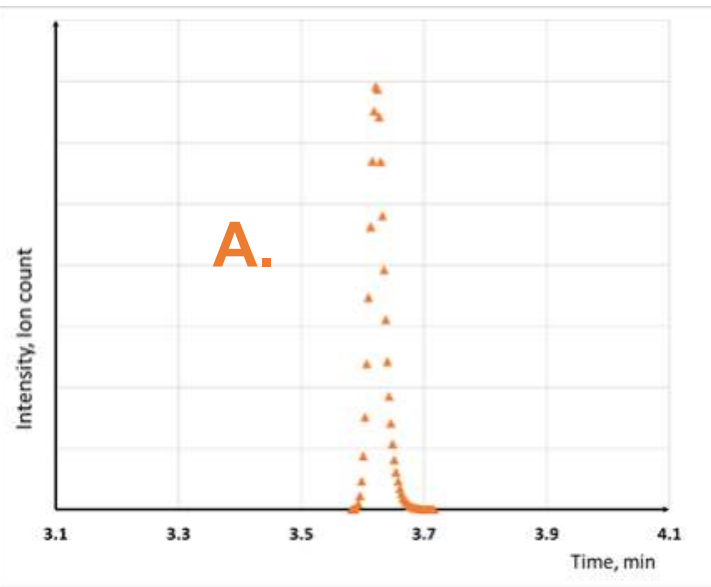

Exponentially Modified Gaussian (EMG):

$$
y=\frac{a c \sqrt{2 \pi}}{2 d} \exp \left(\frac{c^{2}}{2 d^{2}}-\frac{x-b}{d}\right)\left[\frac{d}{|d|}+\operatorname{erf}\left(\frac{x-b}{\sqrt{2} c}-\frac{c}{\sqrt{2} d}\right)\right]
$$

The exponentially modified Gaussian is a convolution of Gaussian and exponential probability density. $a$ = Gaussian heigth, $b=$ location parameter (Gaussian center), $c=$ Gaussian width, $d=$ distortion parameter (a.k.a. modification factor or time constant).

Delley, R. (1985). "Series for the Exponentially Modified Gaussian Peak Shape." Anal. Chem. 57: 388.
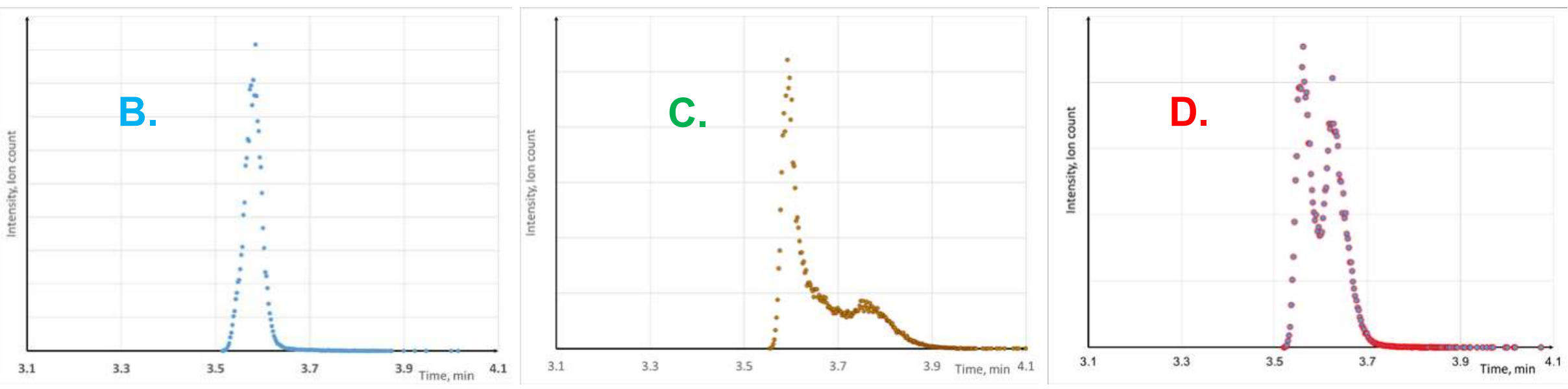

Extracted ion chromatograms peaks for a d5-hippuric acid molecular ion. A. EMG model; B. Successful suitability test; C. Failed suitability test (peak is tailing); D. Failed suitability test (peak is splitting); 


\section{Logarithm transformation}
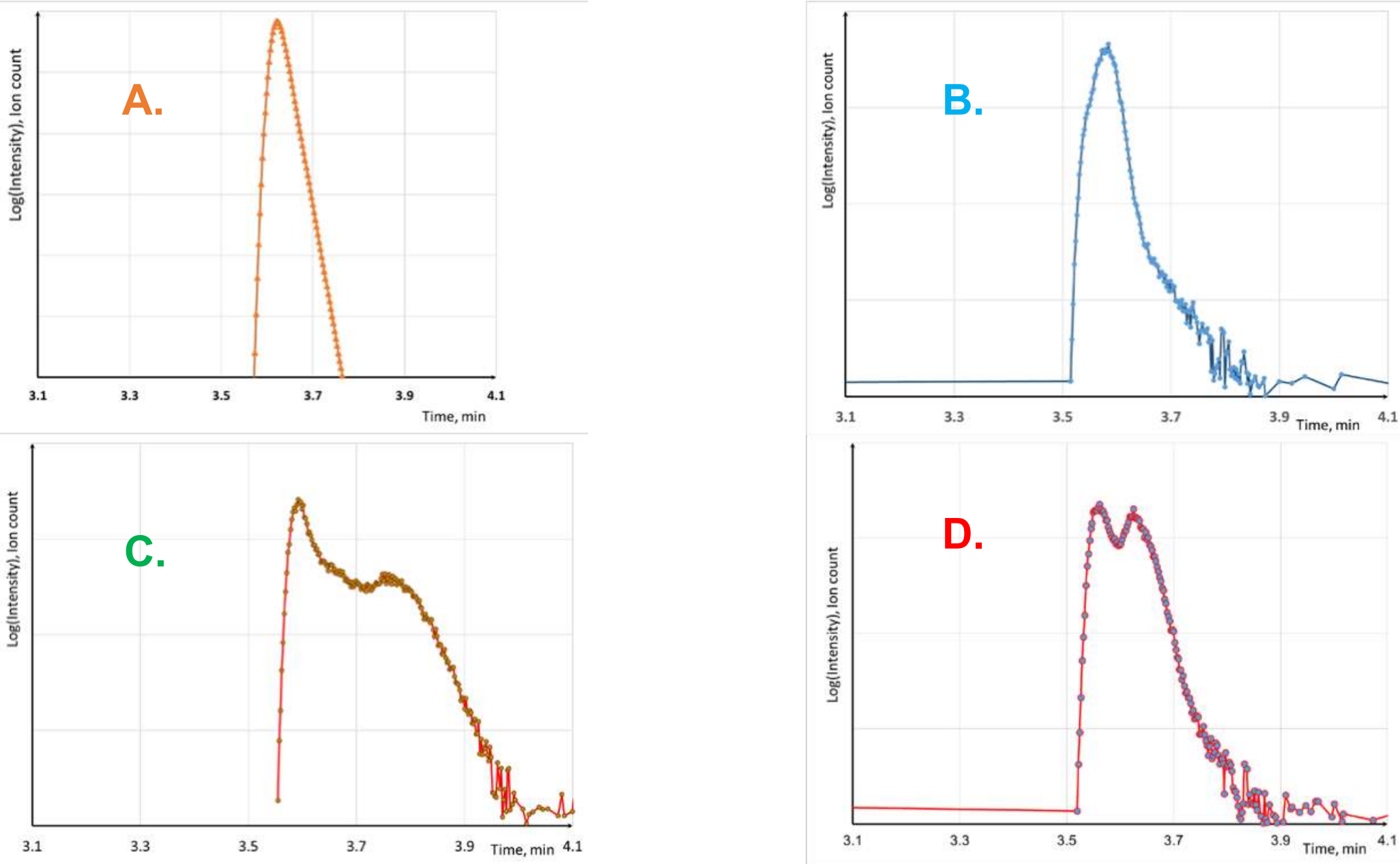

Logarithm transformed extracted ion chromatograms peaks for d5-HA molecular ion: A. EMG model; B. Successful suitability test; C. Failed suitability test (peak is tailing); D. Failed suitability test (peak is splitting); 


\section{Data processing algorithm}

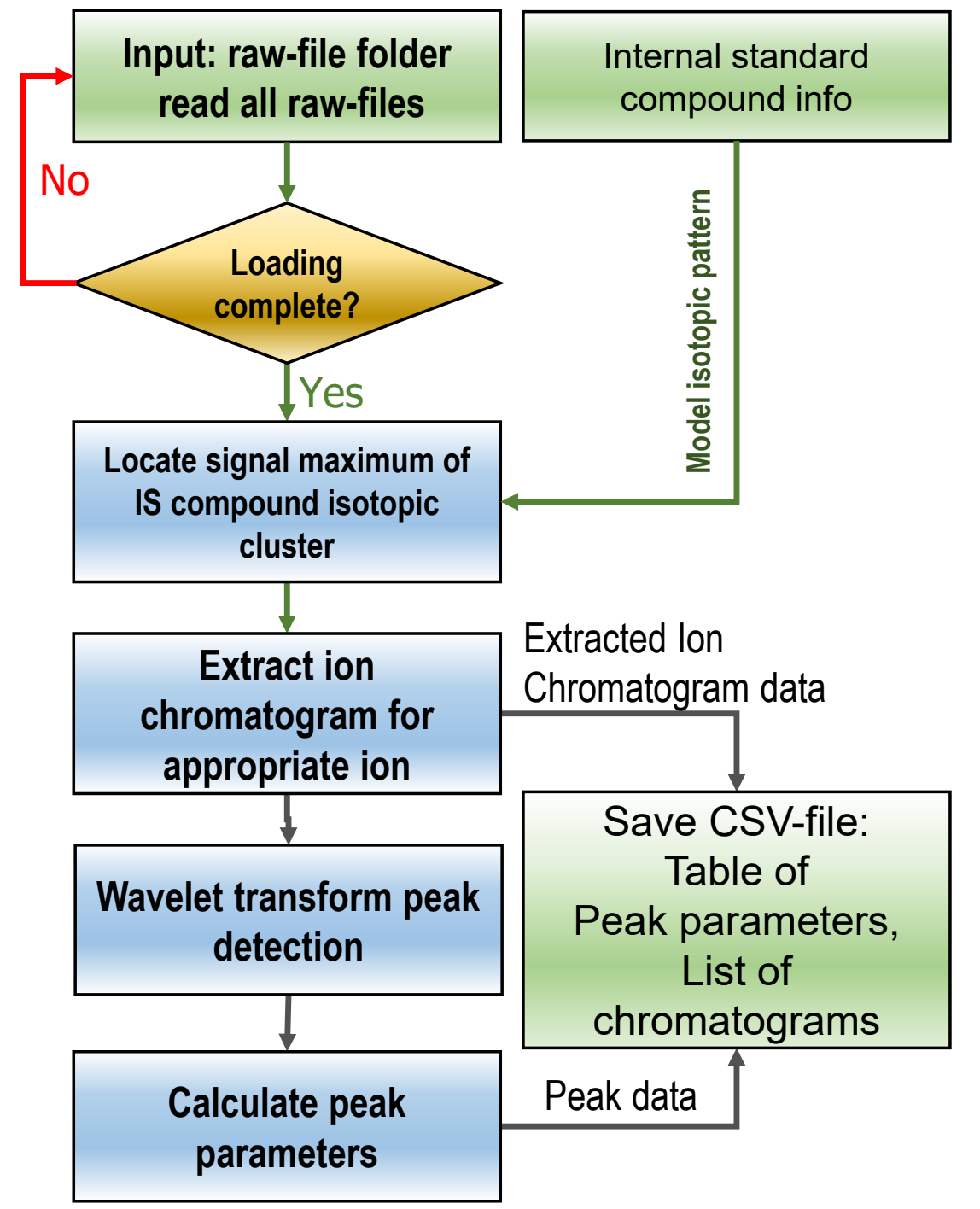

\section{Analyzed IS peaks from Number of projects $=598$ Number of Injections $=58,718$}

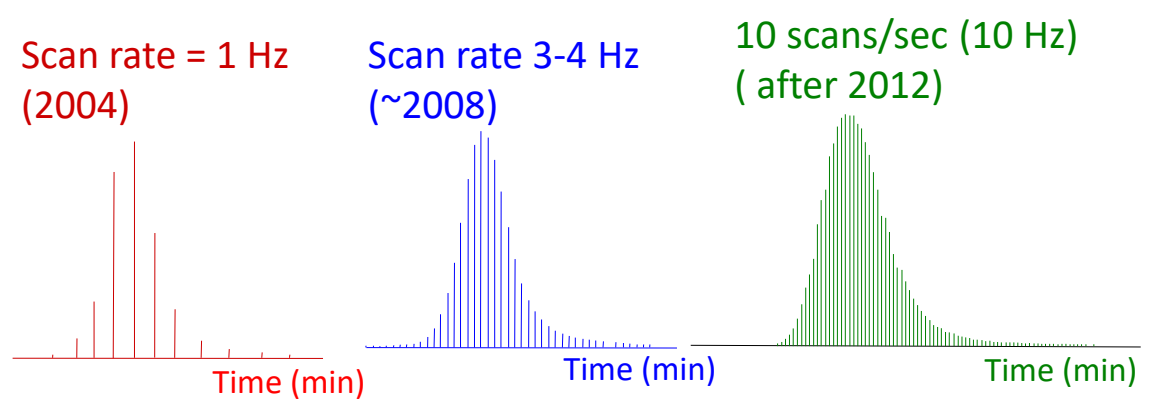

Extracted ion chromatograms for d5-Hippuric acid for various generations of Thermo-Fisher instruments 


\section{Statistics of analysis of IS peaks}

\begin{tabular}{|c|c|c|c|c|}
\hline Peak Parameter & Factors & Average Value* & STDev & Units \\
\hline Position & analyte properties, gradient program & & & \\
\hline start & void volume, & 3.54 & 0.03 & minutes \\
\hline end & & 3.83 & 0.06 & minutes \\
\hline apex & & 3.59 & 0.04 & minutes \\
\hline Width & analyte properties, system settings, & & & \\
\hline $1 / 2$ height & column pluming, gradient program, & 0.1 & 0.02 & minutes \\
\hline $1 / 20$ height & analysis duration & 0.3 & 0.06 & minutes \\
\hline \multicolumn{5}{|l|}{ Half-width } \\
\hline left & & 0.05 & 0.02 & minutes \\
\hline right & & 0.24 & 0.07 & minutes \\
\hline Tailing & stationary phase condition & 1.9 & 0.5 & \\
\hline Height & instrument tune, detector settings & $3.50 E+07$ & $1.30 \mathrm{E}+07$ & counts \\
\hline Area & integration procedure & $1.05 E+08$ & $3.50 E+06$ & counts/min \\
\hline
\end{tabular}

*The average values were calculated for a single multi-day experiment with $\sim 1000$ injections using standard metabolomics protocols and instrument tuning procedures. 


\section{Peak parameters vs. injection order}
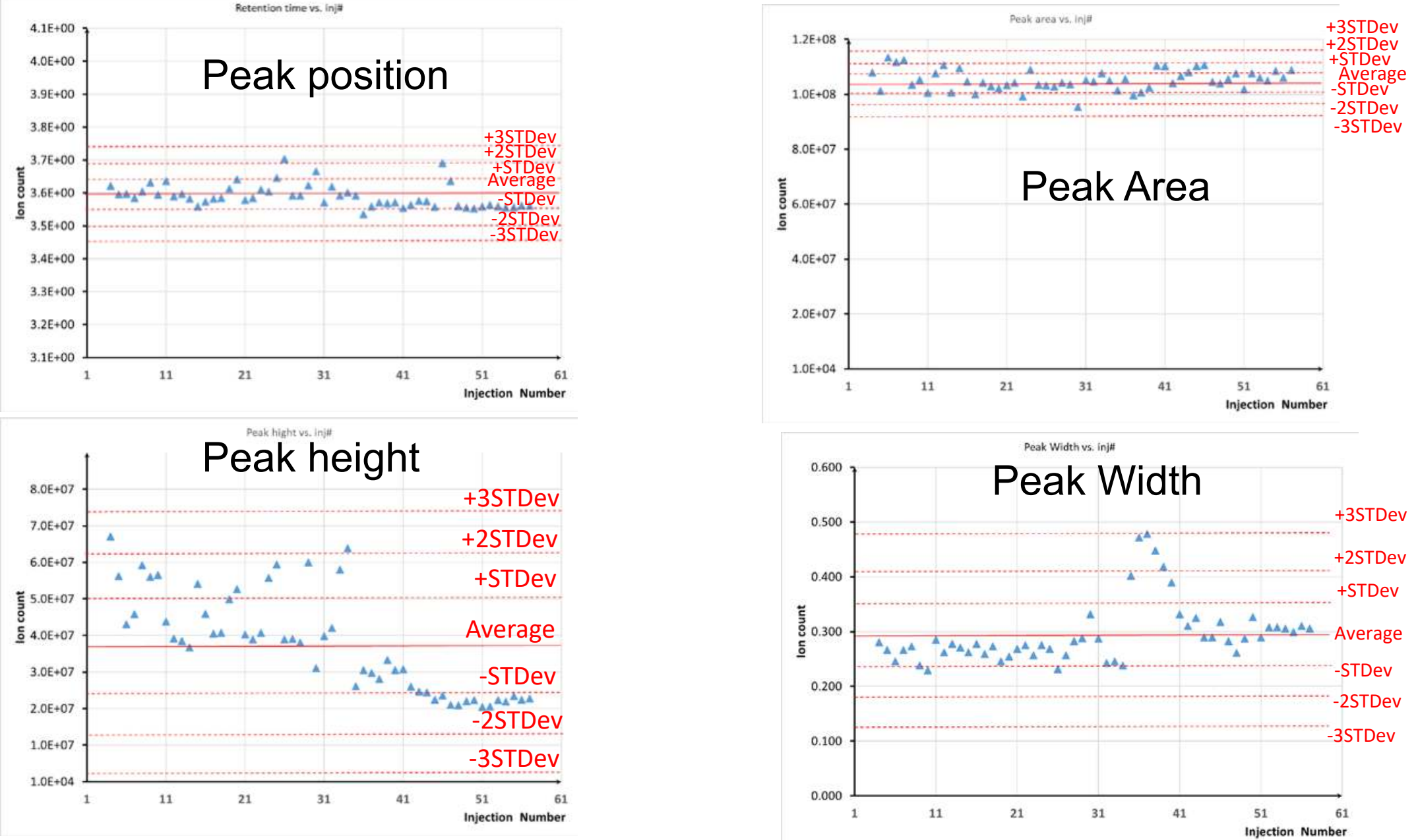

Traditional univariate statistical outlier calculations may be not sufficient to detect early signs of abnormalities in peak parameters. 


\section{Peak data to $28 \times 28$ pixel image}

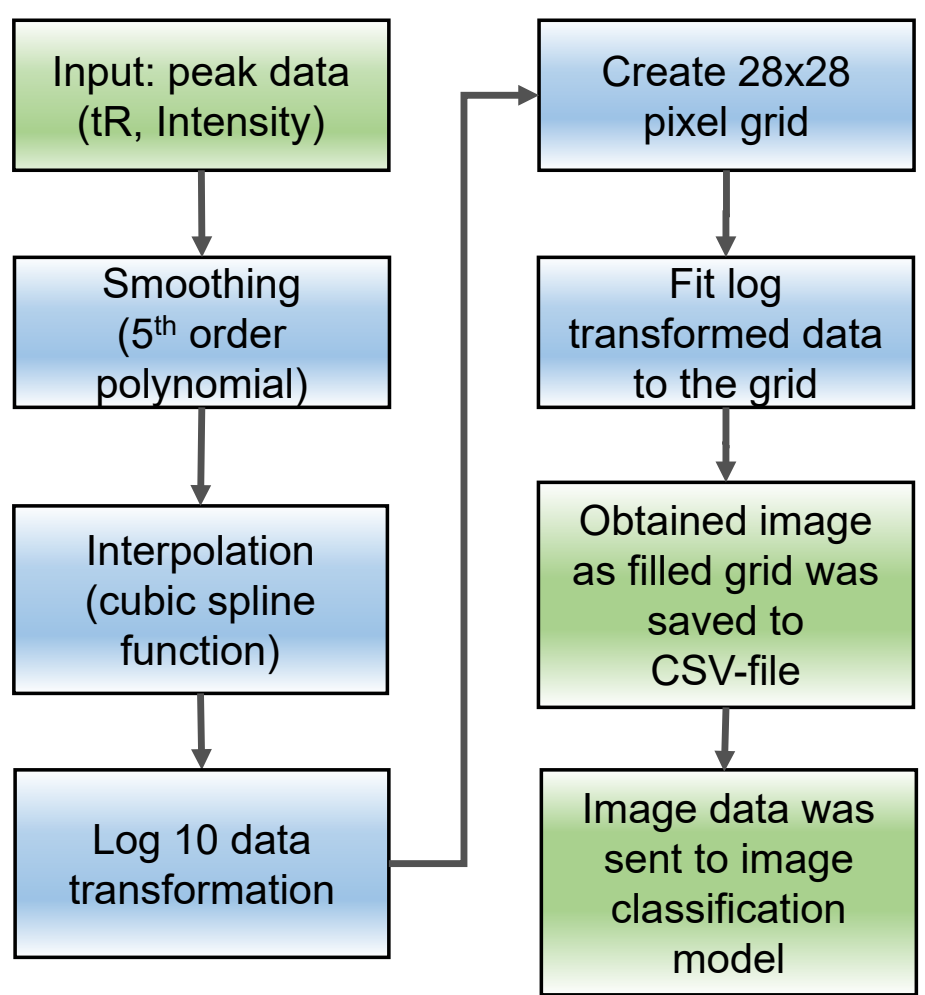

The peak-to-image algorithm includes a logarithmic transformation to take into account a difference of six orders of magnitude between the signal and the background.

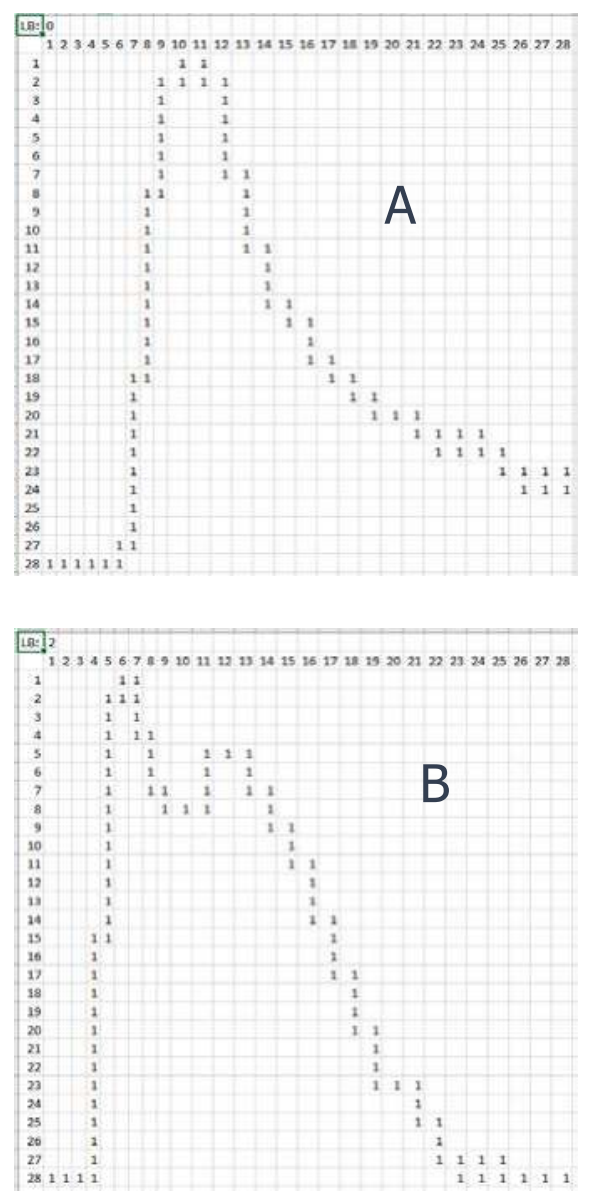

A - suitable

B - abnormal

- $\quad 60,000$ data files were processed

- Manual data review found less than 800 cases of abnormal peaks.

- Two sets of input were created:

- TrainingSet_B1000 .CSV; 500 suitable, 500 abnormal peaks

- TestSet_B300.csv; 150 peak images of each case 


\section{Convolution Neural Network (CNN)}

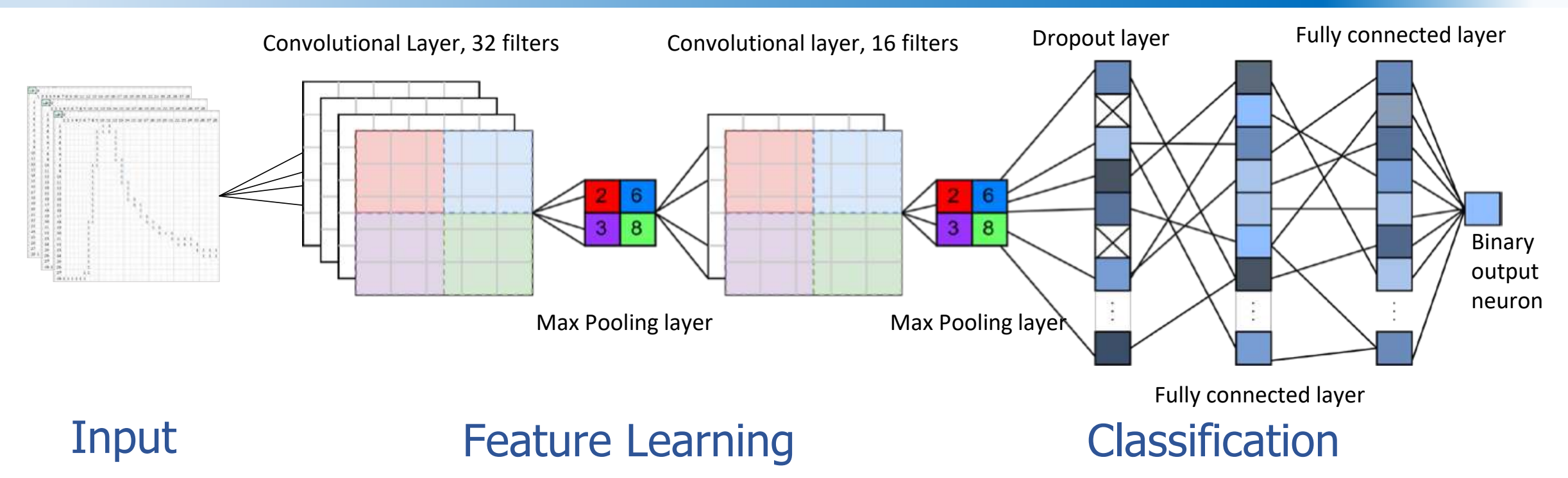

- Convolutional Neural Networks (CNN) excel at detecting shapes, making it suitable for detecting abnormal peaks

- Unlike fully-connected neural networks, CNNs like this one begin with convolutional and pooling (or sub-sampling) layers

$\checkmark$ This makes the CNN more robust to transformations in input, such as slight retention time shifts in peak centroids, and serves as an inbuilt feature extractor

$\checkmark$ This also keeps the total number of trainable free parameters low, even as the input image scales up

- Feature maps synthesized by the convolutional layers are fed into fully connected layers like traditional neural networks, which finally output to a binary sigmoid neuron for classification 


\section{CNN model for peak abnormality detection}

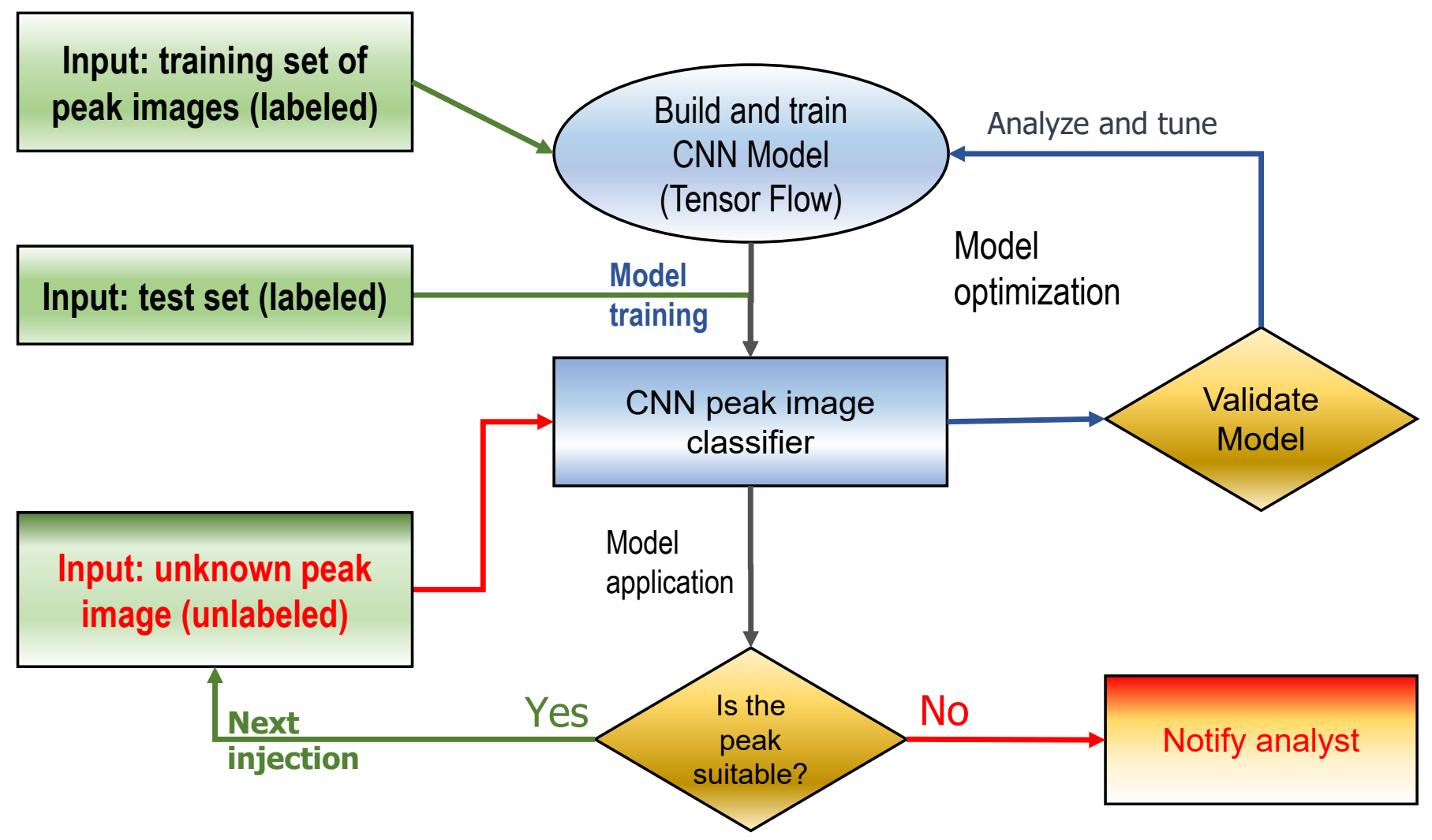

We implemented our CNN model in Python 3.7, using the Keras library and numPy. 


\section{Model prototype implementation using Jupyter Notebook}

- jupyter binary_classifier Last Checkpoint: 01/14/2020 (autosaved)

File Edit View Insert Cell Kernel Help

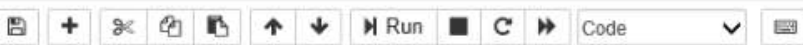

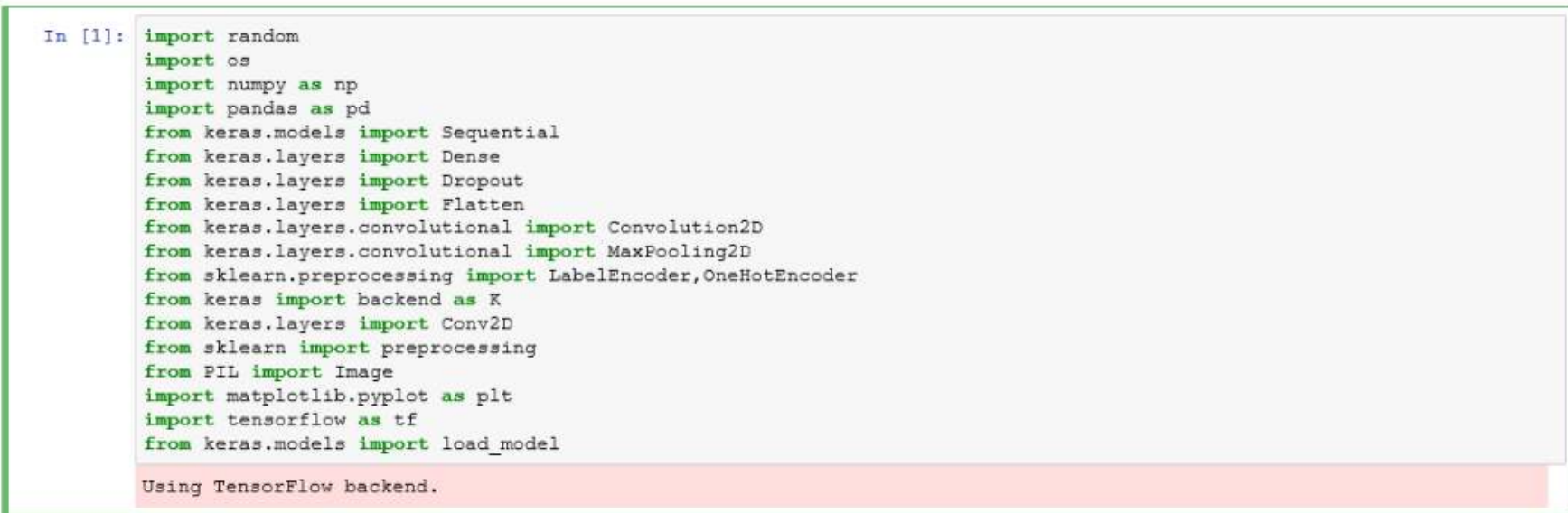

\section{Data Input}

In [2]: \# Load in our test + training data (os agnostic)

testfilepath $=$ os.getcwd ()$+$ os.path.sep + 'Data' + os.path.sep + 'TestSet B300.csv'

trainfllepath $=0$ os.getcwd ()$+0$ s.path.sep + 'Data' + os.path.sep + 'TrainingSet B1000.CSV

test $=$ pd.read_csv (testfilepath, encoding='unicode_escape'). values

train = pd.read csv(trainfilepath, encoding=' unicode escape').values

In [3]: \# Reshape and normalize training dat

trainX = train $[:, 1:]$.reshape (train. shape $[0], 1,28,28$ ) .astype ('float 32 '

$y_{-}$train - train $[:, 0]$

\# Reshape and normalize test data

testX - test $[:, 1:]$.reshape (test. shape $[0], 1,28,28$ ) .astype( 'float 32 '

ytest $=$ test $[:, 0]$ 


\section{Prototype implementation using Jupyter Notebook (continue)}

\section{Prepare labels}

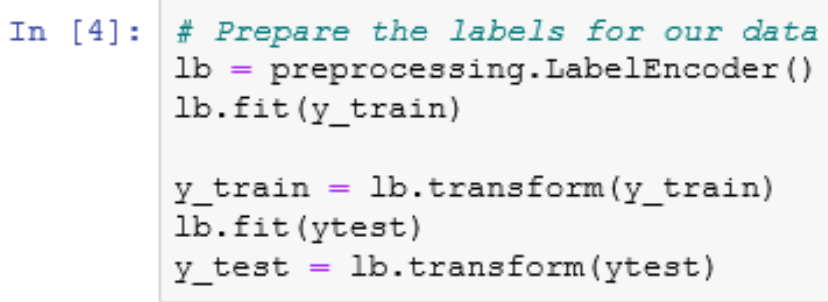

Out [6]: <matplotlib.image.AxesImage at 0x17906c6abe0>

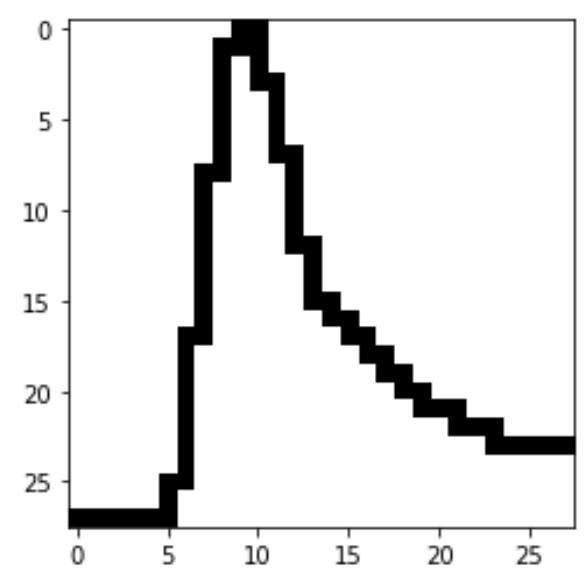

Image of suitable peak

Illı Bristol Myers Squibb 


\section{Prototype implementation using Jupyter Notebook (continue)}

\section{A model based on the LeNet-5 Network [1]}

In [7]: \# binary model

model = Sequential ()

K.set image data format ('channels first') \# corresponds to K. set image dim ordering('th')

model.add (Conv2D $(30,(5,5)$, input shape $=(1,28,28)$, activation= 'relu', border mode= 'valid'))

model .add (MaxPooling2D (pool_size $=(\overline{2}, 2)$ ))

model.add (Conv2D (15, (3, 3), activation= 'relu' ))

model . add (MaxPooling2D (pool size $=(2,2)$ ))

model .add (Dropout (0.2))

model.add (Flatten())

model.add (Dense (128, activation= 'relu' ))

model.add (Dense (50, activation= 'relu' ))

model.add (Dense (1, activation= 'sigmoid'))

\begin{tabular}{|c|c|c|c|}
\hline In $[8]$ : & \multicolumn{3}{|c|}{$\begin{array}{l}\text { \# Compile our model } \\
\text { model.compile (loss='binary_crossentropy', optimizer='adam', metrics= }\end{array}$} \\
\hline \multirow[t]{13}{*}{ In $[9]:$} & \multicolumn{3}{|c|}{$\begin{array}{l}\text { \# Examine the model's parameters } \\
\text { model.summary() }\end{array}$} \\
\hline & \multicolumn{3}{|l|}{ Model: "sequential_1" } \\
\hline & Layer (type) & Output Shape & Param $\neq$ \\
\hline & conv2d_1 (Conv2D) & (None, 30, 24, 24) & 780 \\
\hline & max_pooling2d_1 (MaxPooling2 & (None, 30, 12, 12) & 0 \\
\hline & Conv2d_2 (Conv2D) & (None, 15, 10, 10) & 4065 \\
\hline & $\overline{\max \_p o o l i n g 2 d \_2}$ (MaxPooling2 & (None, 15, 5, 5) & 0 \\
\hline & dropout_1 (Dropout) & (None, 15, 5, 5) & 0 \\
\hline & flatten_1 (Flatten) & (None, 375) & 0 \\
\hline & dense_1 (Dense) & (None, 128) & 48128 \\
\hline & dense_2 (Dense) & (None, 50) & 6450 \\
\hline & $\begin{array}{l}\text { dense } 3 \text { (Dense) } \\
========================\end{array}$ & $\begin{array}{l}\text { (None, 1) } \\
==============:\end{array}$ & 51 \\
\hline & \multicolumn{3}{|c|}{$\begin{array}{l}\text { Total params: } 59,474 \\
\text { Trainable params: } 59,474 \\
\text { Non-trainable params: } 0\end{array}$} \\
\hline
\end{tabular}

Train Model

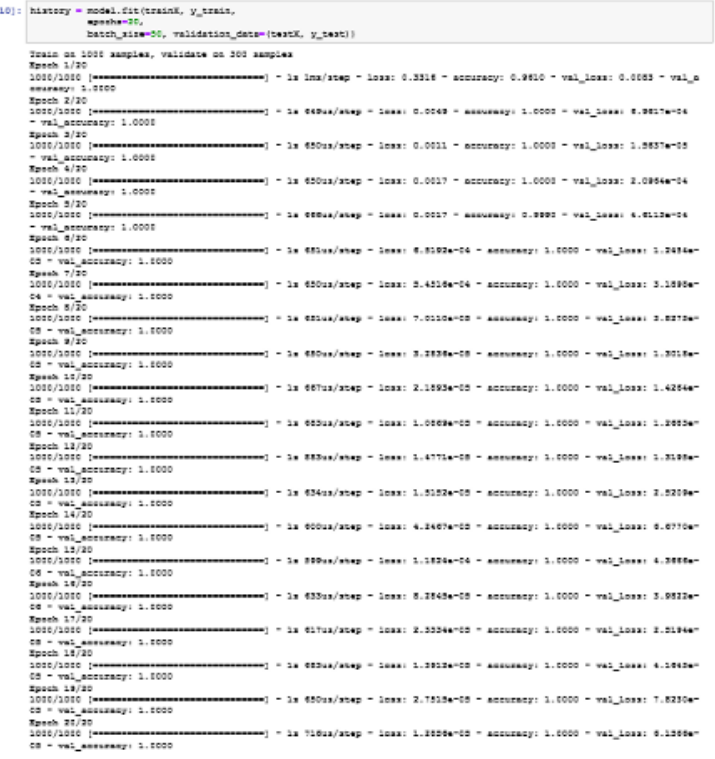

Training takes $\sim 15$ seconds (Model trained on CPU; Intel I5-8350; 8Gb RAM) 


\section{Prototype implementation using Jupyter Notebook (continue)}

\section{Model perfomance evaluation}

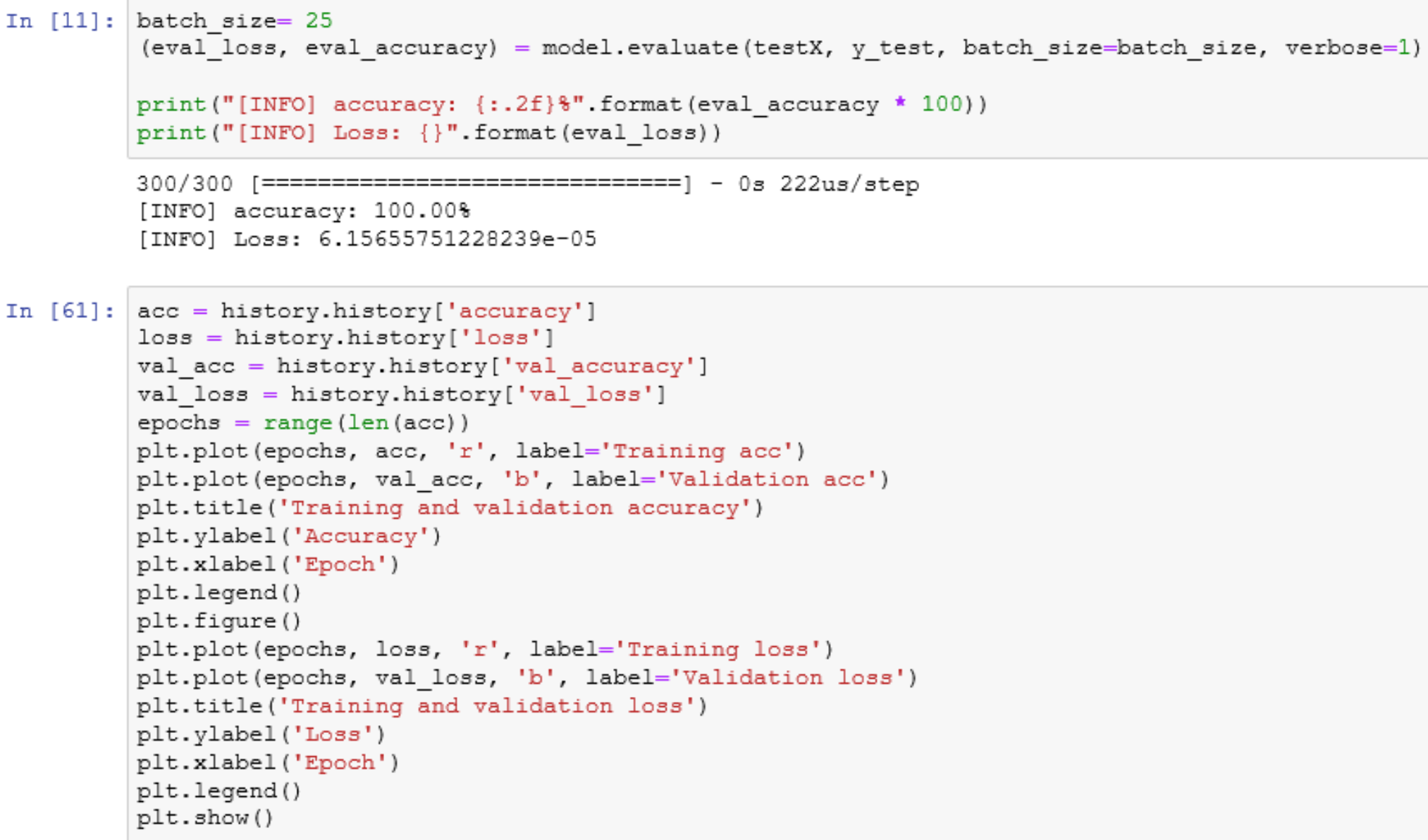




\section{Prototype implementation using Jupyter Notebook (continue)}
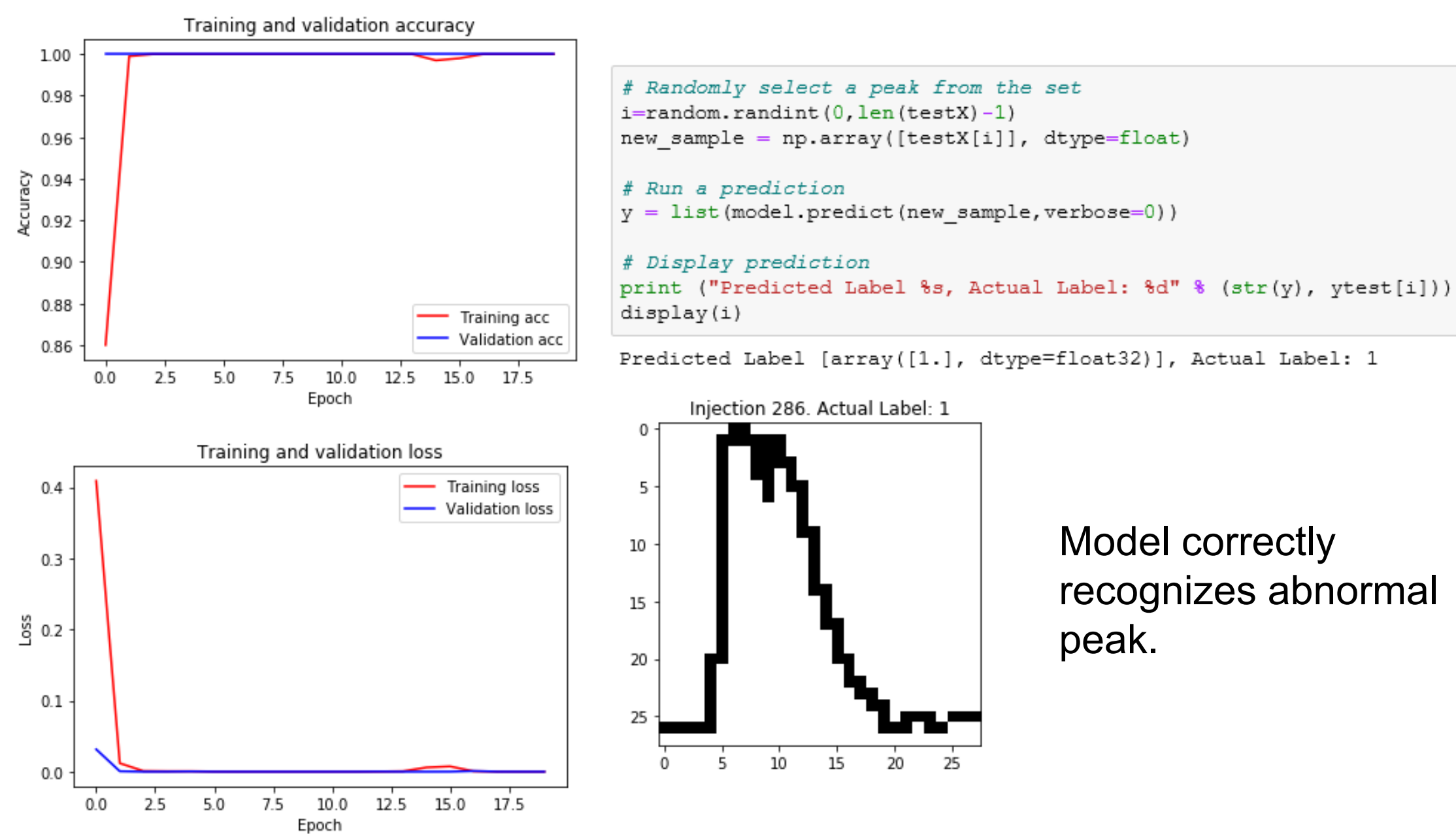

Model correctly recognizes abnormal peak. 


\section{Continuous injection quality monitoring}

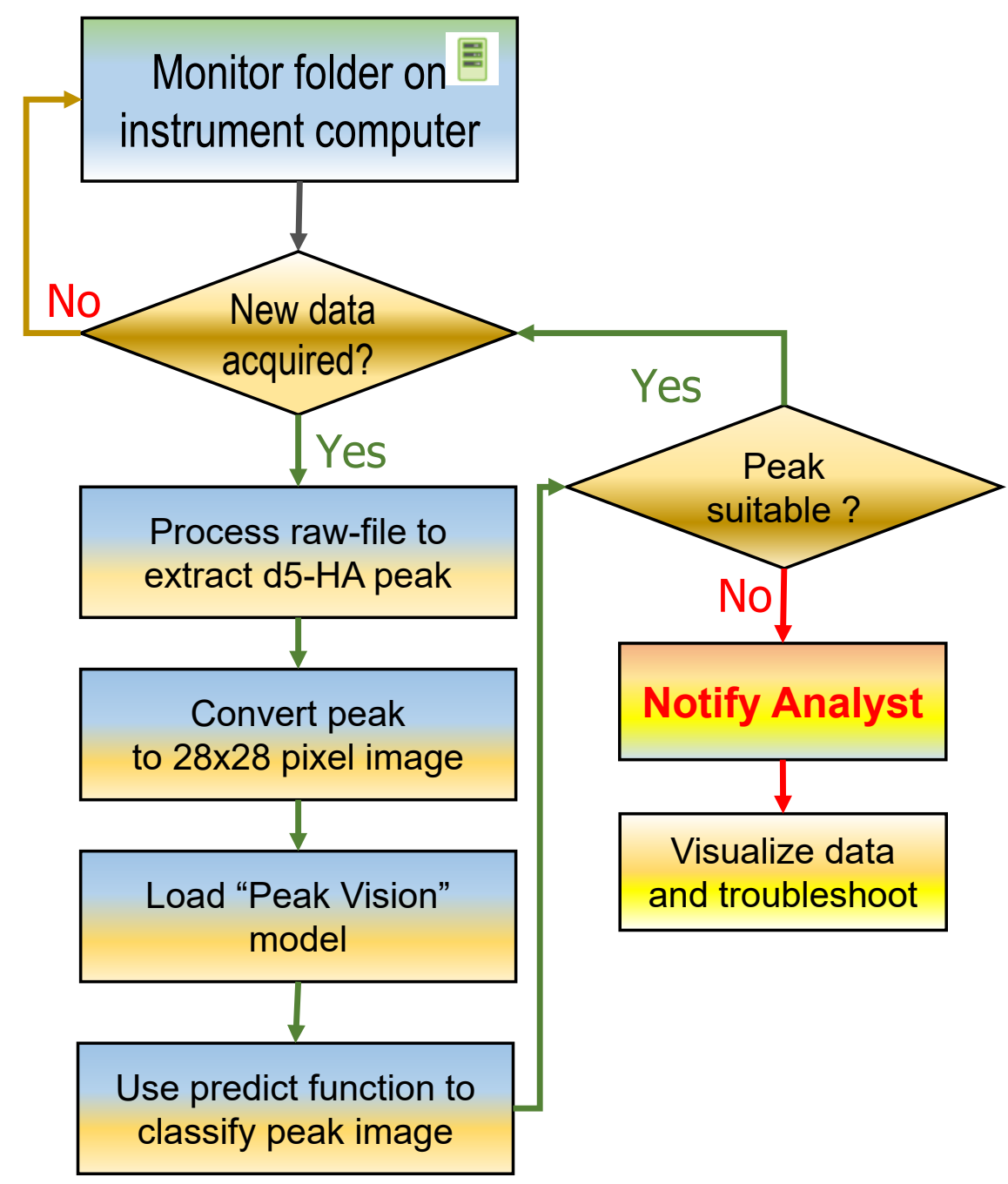

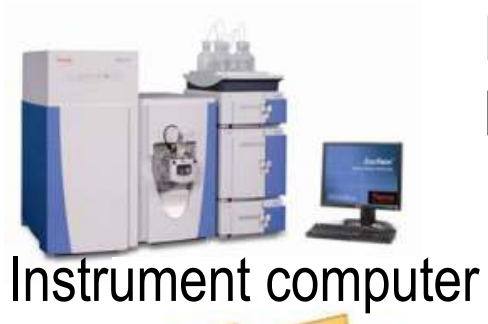

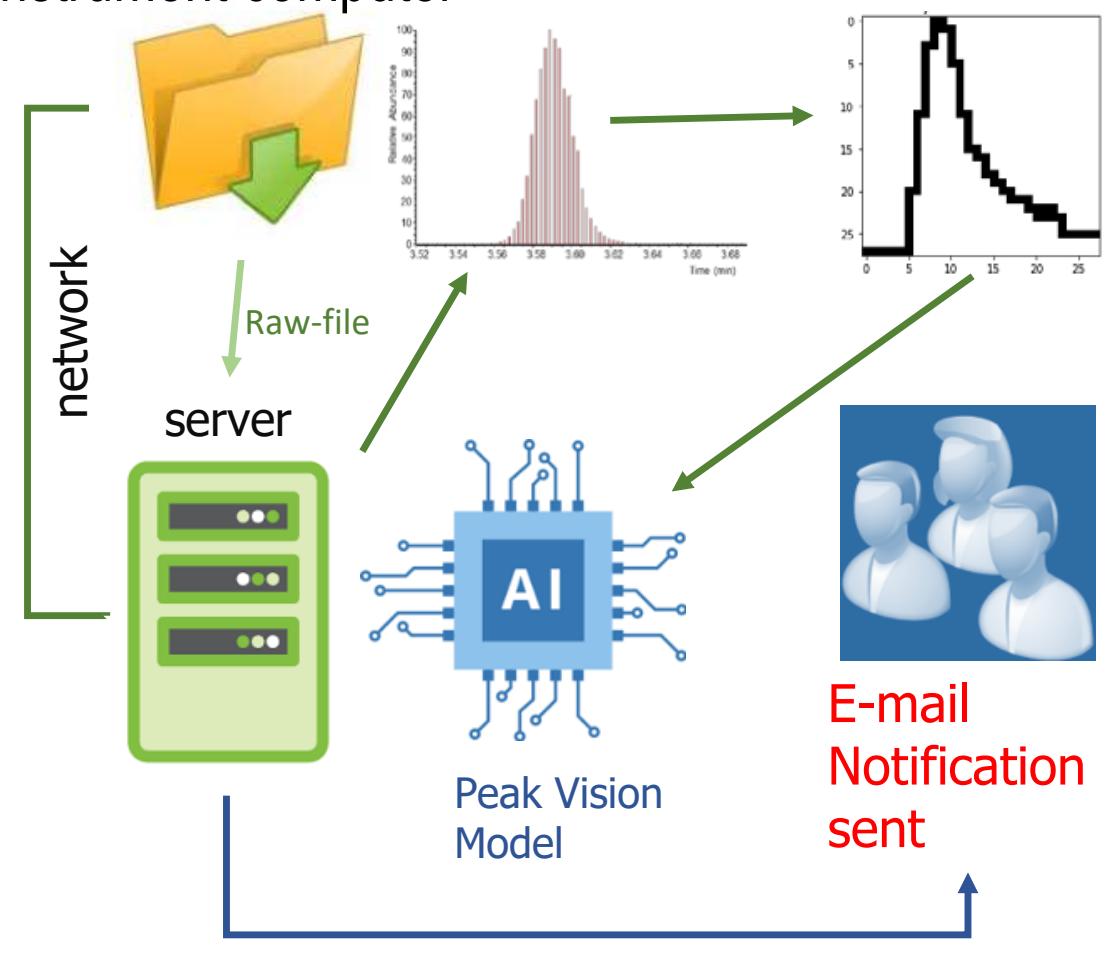

Fully tuned and calibrated HR LC-MS system
ACS Spring 2020 National Meeting \& Expo 


\section{Example \#1 experiment monitoring with CNN (monitoring run was performed post data collection)}

Project: metabolomics analysis of human serum samples from 400 patients comprised of healthy volunteers and patients diagnosed with nonalcoholic steatohepatitis (NASH). A total of five 96- well plates including interpolate quality controls and blanks were used.

Analysis took $\sim 5$ days per polarity.

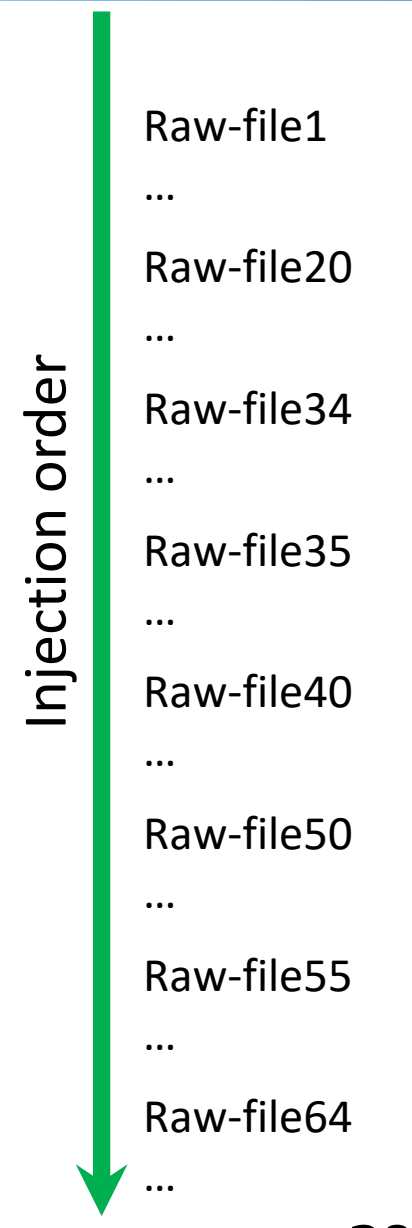

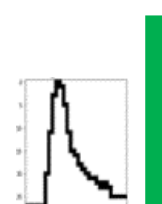
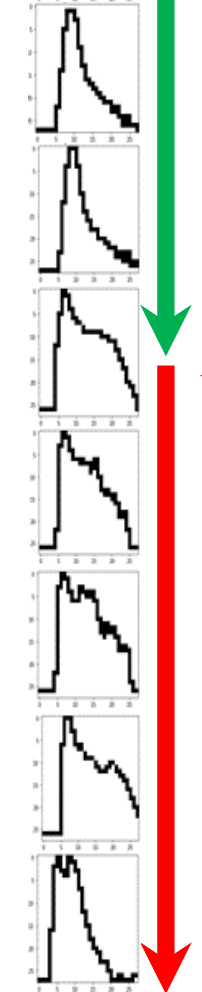

CNN model

suitable

suitable

abnormal

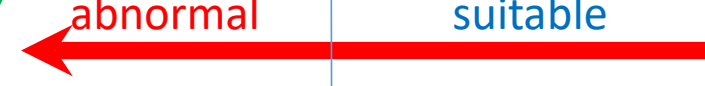

abnormal

abnormal

abnormal

abnormal

3435
Analyst review

suitable

suitable

suitable

suitable

abnormal

abnormal

abnormal

abnormal
Visual detection by human w/o log transform

The model detected the abnormality 29 injections (425 min) before system failure

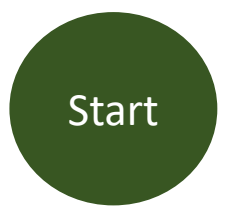

(Ill Bristol Myers Squibb 


\section{Example \#2 experiment monitoring with CNN}

Restarted system. After cleaning, recalibrating and tuning system with new model

1

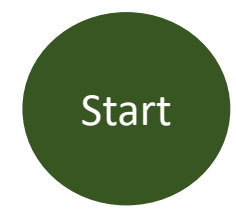

Illı Bristol Myers Squibb

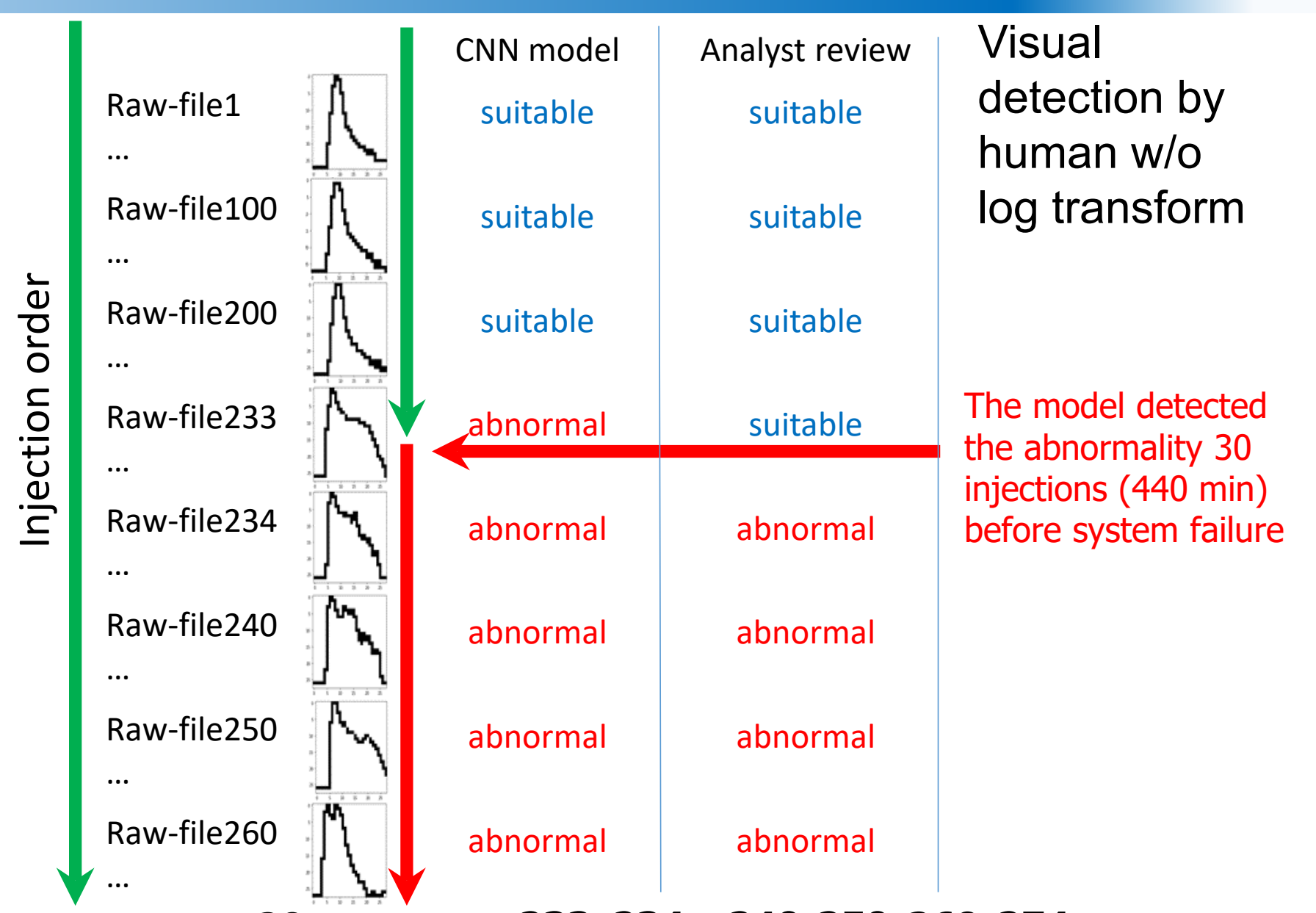

233234240250260274

\section{0

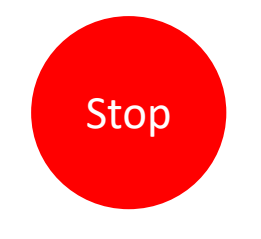




\section{Example \#3 experiment monitoring with CNN}

Restarted system again. Cleaning, recalibrating and tuning system with new model

1

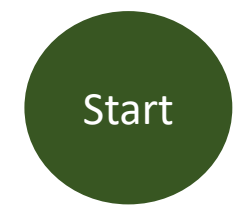

Ill' Bristol Myers Squibb

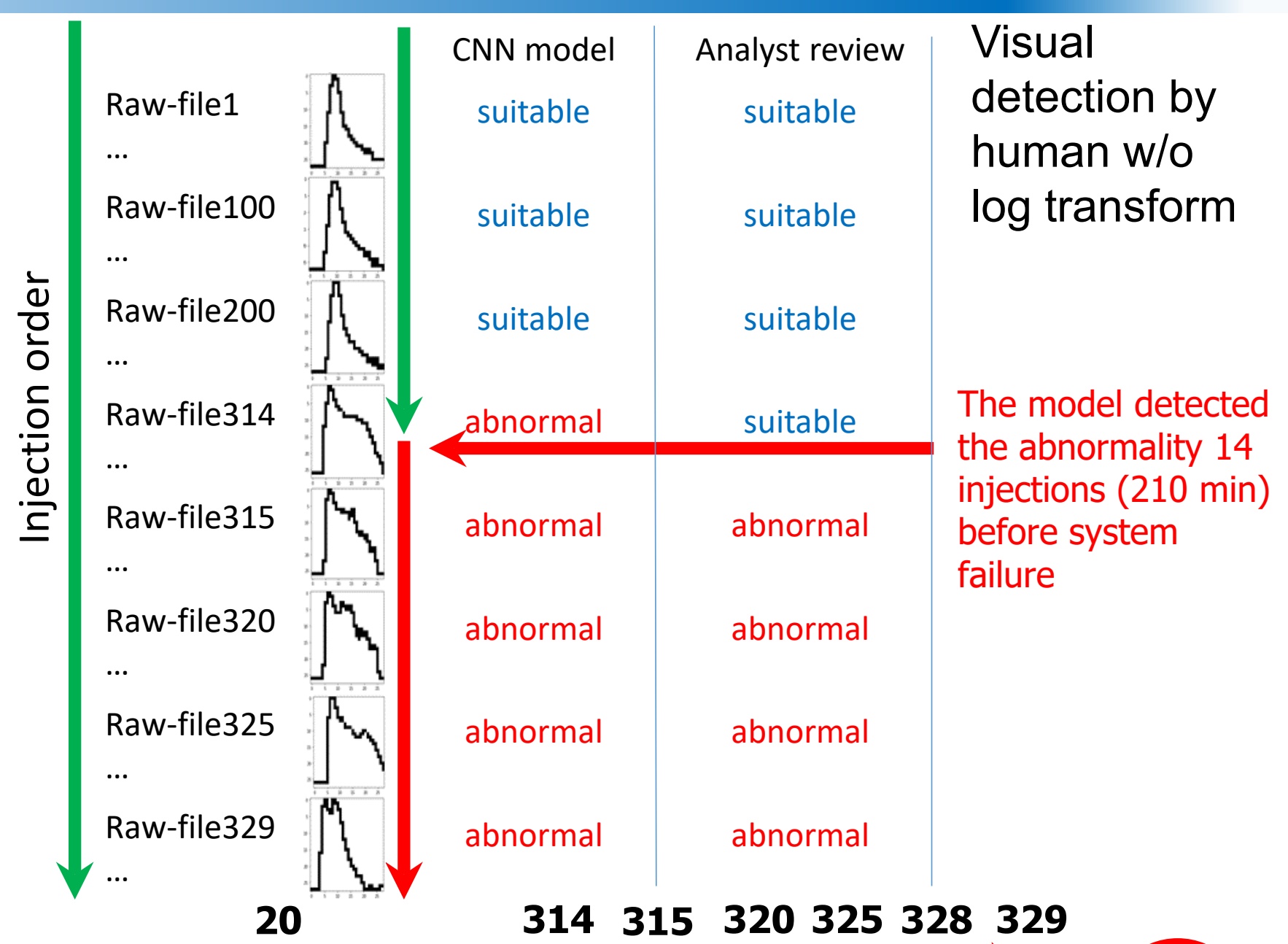

314315320325328329

\section{0

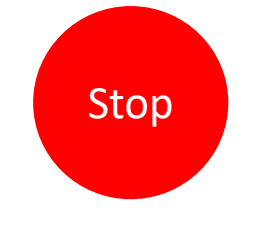




\section{Lessons learned}

- Maintaining the data archives of an analysis, including its raw-files, should become standard operating procedure. Historical data is used for data mining, the optimization of models and the testing of new ideas. Pattern recognition algorithms need data from both successful and failed experiments.

- CNN-based models are excellent tools for detecting patterns in data, as well as abnormal cases.

- A major obstacle with the creation of a CNN-based model was the need for a large training set. In our experience, cases of failed runs were rare.

- Our HR-LC-MS monitoring application enables the automated review of all built-in experiment quality controls during the experiment which can potentially save precious samples 


\section{Conclusions \& Future Work}

\section{Current Solution}

- Implements a non-intrusive, non-disruptive, machine-learning-based continuous system suitability monitoring algorithm

- Able to detect abnormalities $\sim 20$ injections (300 minutes) before the system shuts down e.g. due to excessive back pressure

\section{Further Investigation}

- Change data retention policy, data from "failed" runs are often not saved, but needed for training accurate models

- Fine-tune peak classification, expand with known shapes to improve troubleshooting of instrument or sample based issues

- Test higher image resolutions, network configurations, etc.

\section{Future Expansions}

Further work directions include an investigation into the possibility of monitoring a mixture of stable label standards, which would increase the resolution of the data and allow us to test the stability of the model.

- Extend system to work with a variety of LC-MS systems

- Extend system with multiple stable label standards

- Extend for LC-MS/MS experiments

- Implement cloud-based solutions 


\section{Acknowledgements}

Pharmaceutical Candidate Optimization

Discover the right molecule, enable the clinical plan

Michael Reily

- Petia Shipkova

Lois Lehman-McKeeman

- Colleen McNaney

Matthew Cintron

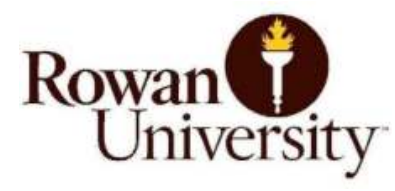

- Dr. Vasil Hnatyshin, Computer Science

- Dr. Umashanger Thayasivam, Mathematics

Joseph Scavetta, Bioinformatics 